\title{
Retraso en el inicio del tratamiento en pacientes con tuberculosis resistente a fármacos
}

\section{Delayed initiation of treatment in patients with drug-resistant TB}

\author{
Miroslava Félix-Ponce, ${ }^{*}$ Alejandra Isabel Jiménez-Gracia,* Rafael Laniado-Laborín*,‡
}

*Universidad Autónoma de Baja California;

${ }^{\ddagger}$ Hospital General de Tijuana. Sistema Nacional de Investigadores, CONACYT.

\begin{abstract}
RESUMEN. Introducción: El inicio oportuno del tratamiento es crucial para la gestión programática en los países con una alta carga de tuberculosis multidrogorresistente (TB-MDR). El retraso en el inicio del tratamiento permite la transmisión en la comunidad y la aparición de casos de tuberculosis multidrogorresistente. Material y métodos: Se analizó el archivo de la Clínica y Laboratorio de Tuberculosis del Hospital General Tijuana y se incluyeron todos los casos con diagnóstico de tuberculosis con resistencia a rifampicina o multidrogorresistente durante el período comprendido entre el año 2006-2019. Se determinó el intervalo en días entre el dictamen clínico y el inicio de tratamiento. Resultados: Se incluyeron 52 pacientes. El retraso en el inicio de tratamiento después de haber dictaminado el caso como TB-RR/MDR fue de $115.6 \pm$ 109.2 días (3.8 meses) con una mediana de 97.5 días. El retraso en los pacientes que egresaron por curación fue de 113.4 días versus 155.0 para los pacientes que fallecieron $(p=0.83)$. Conclusiones: $\mathrm{El}$ intervalo de tiempo entre el dictamen de un caso de tuberculosis resistente a rifampicina/multidrogorresistente y el inicio del tratamiento (115 días) es inaceptablemente prolongado. La red para la atención de la tuberculosis multidrogorresistente se vería fortalecida con la descentralización de las existencias de fármacos antituberculosis de segunda línea.
\end{abstract}

Palabras clave: Multidrogorresistencia, retraso, tratamiento, tuberculosis.

\section{INTRODUCCIÓN}

La tuberculosis multidrogorresistente (TB-MDR) es uno de los principales retos para los programas de control de lucha contra la tuberculosis (TB) y un importante obstáculo para alcanzar las metas de la estrategia Fin de la Tuberculosis de la Organización Mundial de la Salud (OMS). El diagnóstico precoz y el inicio oportuno del tratamiento son cruciales para la gestión programática en los países con una alta carga de TB-MDR. El retraso en el inicio del tratamiento

Correspondencia:

Dr. Rafael Laniado-Laborín

Hospital General de Tijuana, Tijuana, Baja California.

Correo electrónico: rlaniado@uabc.edu.mx

Trabajo recibido: 17-VII-2019; aceptado: 07-XI-2019.
ABSTRACT. Introduction: Timely initiation of treatment is crucial for program management in countries with a high burden of multidrugresistant tuberculosis (MDR-TB). The delay in the initiation of the treatment allows the transmission in the community and the appearance of TB-MDR cases. Material and methods: Retrospective review of the files of the Tuberculosis Clinic of the Tijuana General Hospital, including all cases with a diagnosis of tuberculosis with rifampicin resistance (RRTB) or MDR-TB, during the period between 2006-2019. The interval (in days) between the regimen prescription and the start of treatment was determined. Results: 52 patients were included. The delay in the initiation of treatment after having recommended a treatment regimen for TB-RR /MDR was $115.6 \pm 109.2$ days (3.8 months) with a median of 97.5 days. The delay in patients who were cured was 113.4 days versus 155.0 days for patients who died $(p=0.83)$. Conclusions: The time interval between prescription of treatment of an RR/MDR-TB case and the start of treatment (115 days) is unacceptably long. The network for MDR-TB care would be strengthened with the decentralization of stocks of second-line anti-tuberculosis drugs.

Keywords: Multidrug resistance, delay, treatment, tuberculosis.

permite la transmisión en la comunidad y la aparición de casos emergentes de TB-MDR. ${ }^{1}$

A pesar de que la ampliación de la capacidad de diagnóstico y la atención centrada en el paciente podrían mejorar la vinculación entre el diagnóstico de TB-MDR y el inicio del tratamiento, un problema persistente en los países de ingresos bajos a medios es el de pacientes con TB-MDR confirmada, en lista de espera para iniciar el tratamiento. ${ }^{2}$

A pesar de los esfuerzos para combatir la TB-MDR, la mayoría de los pacientes en los países de alta carga experimentan retrasos de meses (incluso de años) en el inicio del tratamiento por fallas en el sistema de salud. ${ }^{3}$

$\mathrm{Al}$ experimentar persistentemente retrasos en el inicio del tratamiento después de haber dictaminado y asignado un esquema de fármacos para nuestros pacientes, decidimos revisar nuestra base de datos de pacientes 
Neumol Cir Torax. 2019; 78 (4): 363-365

con tuberculosis resistente a rifampicina o rifampicina e isoniacida (TB-RR/MDR) y determinar el grado de retraso de manera objetiva.

\section{MATERIAL Y MÉTODOS}

Se analizó el archivo de la Clínica y Laboratorio de Tuberculosis del Hospital General Tijuana y se incluyeron todos los casos con diagnóstico de TB-RR/MDR durante el período comprendido entre el año 2006-2019. Se obtuvo información demográfica, historia de tratamientos previos, comorbilidades, resultados de microbiología, intervalo en días entre el dictamen clínico y el inicio de tratamiento, esquemas de tratamiento y resultados de micobacteriología.

En todos los pacientes se identificó al complejo $M$. tuberculosis, inicialmente mediante Accuprobe y posterior por cromatografía lateral (SD TB Ag MPT $64^{\circledR}$ ). En todos los casos el perfil de resistencia a isoniacida y rifampicina se obtuvo a través de cultivo líquido (MGIT 960, Becton Dickinson $\left.^{\circledR}\right)$; a partir del año 2014 se realizó, además, la prueba de Xpert MTB/RIF (Cepheid Inc ${ }^{\circledR}$ ) como tamizaje en todos los grupos de riesgo para TB drogorresistente.

Para el análisis de la información se utilizó el paquete comercial SPSS ${ }^{\circledR}$ Ver. 24. Se calcularon los estadísticos descriptivos y se utilizó una prueba de ANOVA para comparar las medias en el retraso en el inicio del tratamiento en relación con el estado clínico, y una correlación bivariada entre el retraso en el inicio del tratamiento y el tiempo requerido para la conversión del cultivo. La región crítica se fijó en $<0.05$.

\section{RESULTADOS}

Se incluyeron 52 pacientes, 30 de ellos del sexo masculino (57.7\%), con una edad media de $39.6 \pm 16.8$ años (rango 13 a 76 años). Veintiocho pacientes (53.8\%) presentaban alguna comorbilidad, siendo la diabetes con 16 casos (30.8\%) la más frecuente; cuatro pacientes eran hipertensos $(7.7 \%)$, tres pacientes presentaban coinfección con virus de hepatitis C (5.8\%) y dos pacientes coinfección por VIH (3.8\%).

Dieciocho pacientes (34.6\%) eran casos nuevos, 20 pacientes $(38.5 \%)$ habían recibido un tratamiento previamente, cuatro $(7.7 \%)$ habían recibido tratamiento en dos ocasiones, siete $(13.5 \%)$ en tres ocasiones y tres pacientes (5.8\%) habían recibido tratamiento antituberculosis en cuatro ocasiones.

El retraso en el inicio de tratamiento después de haber dictaminado el caso como TB-RR/MDR fue de $115.6 \pm 109.2$ días (3.8 meses) con una mediana de 97.5 días (cuartil 25\%: 43.5 días; cuartil 75\%: 138.5 días). La Tabla 1 muestra el retraso del tratamiento en relación con el estado del paciente. El retraso en los pacientes que egresaron por curación fue de 113.4 días versus 155.0 para los pacientes que fallecieron $(p=0.83)$; en la Figura 1 se muestra el retraso anual en el inicio del tratamiento.

El tiempo de conversión del cultivo para el grupo fue de $92.2 \pm 56.7$ días después de haber iniciado el trata-

Tabla 1: Intervalo entre dictamen e inicio de tratamiento de acuerdo con la condición clínica del paciente.

\begin{tabular}{|l|c|c|}
\hline \multicolumn{1}{|c|}{ Condición } & Pacientes $(\mathbf{n})$ & Intervalo (días) \\
\hline Curado & 31 & 113.4 \\
\hline Defunción & 7 & 155.0 \\
\hline En tratamiento & 9 & 104.2 \\
\hline $\begin{array}{l}\text { Pérdida de } \\
\text { seguimiento }\end{array}$ & 3 & 118.3 \\
\hline Traslado & 2 & 59.5 \\
\hline
\end{tabular}

Días transcurridos entre el dictamen e inicio del tratamiento

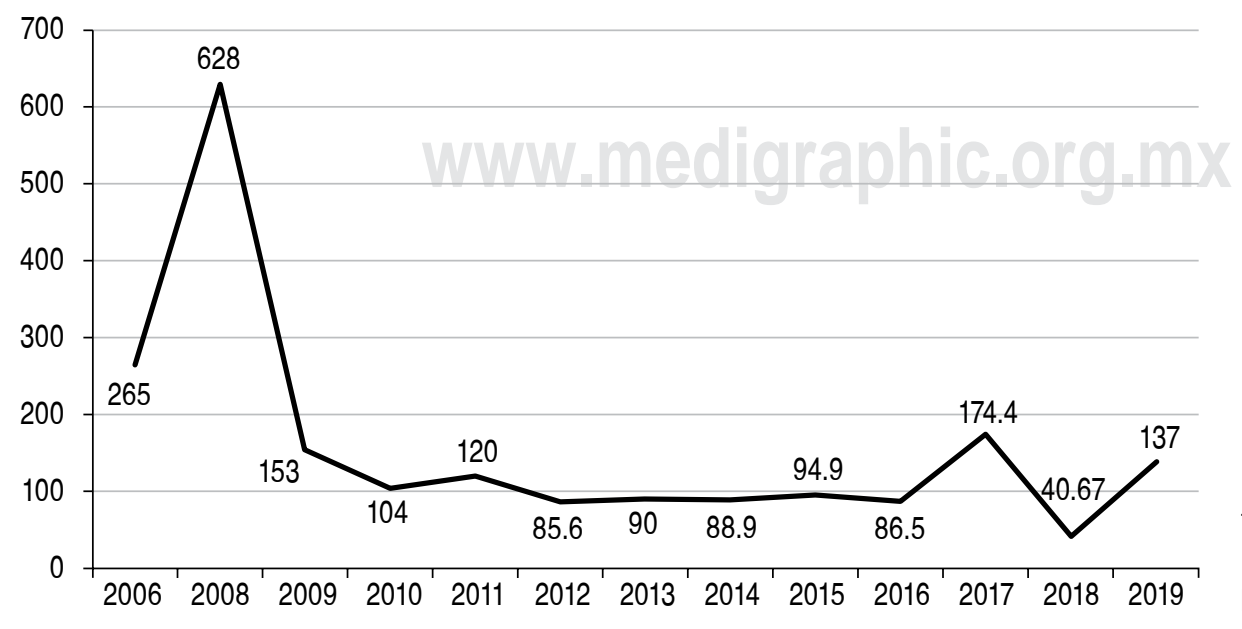

Figura 1:

Intervalo de días entre el dictamen de un caso y el inicio del tratamiento para tuberculosis resistente a rifampicina 0 rifampicina e isoniacida (TB RR/MDR) por año (2006-2019). 
miento. La correlación bivariada entre el retraso en el inicio del tratamiento y la conversión del cultivo mostró una correlación positiva débil ( $r$ de Pearson: 0.288; $p$ $=0.06$ ).

\section{DISCUSIÓN}

En países con una alta carga de TB-MDR, y México es el tercer país con mayor carga de TB-MDR en las Américas, los retrasos en el inicio del tratamiento constituyen un desafío para alcanzar los objetivos de la estrategia Fin a la Tuberculosis de la Organización Mundial de la Salud. ${ }^{4}$

A pesar del control progresivo de la TB a nivel global, la carga de la enfermedad entre los pacientes con TB-MDR se ha mantenido prácticamente sin cambios. Los retrasos en el inicio del tratamiento tienen un impacto a nivel individual, al favorecer el progreso de la enfermedad; $y$ a nivel comunitario, al prolongar la transmisión de la TB-MDR en la comunidad. La atención eficaz de la TB-MDR se basa, entre otras cosas, en el acceso temprano al tratamiento; sin embargo, los retrasos en el inicio de éste, después de haber establecido el diagnóstico, constituyen un problema no resuelto. ${ }^{5}$

Los retrasos en el inicio del tratamiento para TB-RR/ MDR reportados en la literatura van desde un mínimo de cinco días hasta un máximo de 105 días..$^{5-9}$

El intervalo de tiempo en nuestro medio, entre el dictamen de un caso TB-RR/MDR y el inicio del tratamiento (115 días), es inaceptablemente prolongado y secundario exclusivamente al retraso en la disponibilidad del esquema de tratamiento, como consecuencia de un trámite burocrático ineficiente a nivel local o al desabasto de fármacos a nivel nacional. Además de prolongar el período de transmisibilidad hacia la comunidad de un caso de TB resistente a fármacos, encontramos una tendencia a mayor retraso en la conversión del cultivo a mayor retraso en el inicio del tratamiento, y aun cuando no alcanzó significancia estadística, el retraso en el inicio del tratamiento fue 42 días más prolongado en los pacientes que fallecieron que en los pacientes que egresaron por curación; este hallazgo ya ha sido reportado en la literatura. 5,10

La introducción del Xpert MTB/RIF, método molecular rápido para la identificación de Mycobacterium tuberculosis y resistencia a rifampicina, fue diseñado para reducir los retrasos en el diagnóstico, ya que permite obtener resultados en menos de dos horas; de hecho, este método ha sido recomendado por la OMS como método de tamizaje inicial en todos los pacientes con sospecha de TB-RR/ MDR. ${ }^{11}$ Sin embargo, aun este método de diagnóstico rápido no influye sobre el retraso en inicio del tratamiento posterior al dictamen.

\section{CONCLUSIONES}

La red para la atención de la TB-MDR se vería fortalecida con la descentralización de las existencias de los fármacos antituberculosis de segunda línea, ${ }^{5}$ donde cada estado tendría un número determinado de tratamientos de acuerdo con su incidencia de casos TB-RR/MDR para inicio inmediato del tratamiento, mismos que se repondrían conforme se fueran utilizando.

\section{REFERENCIAS}

1. Htun YM, Khaing TMM, Yin Y, et al. Delay in diagnosis and treatment among adult multidrug resistant tuberculosis patients in Yangon Regional Tuberculosis Center, Myanmar: a cross-sectional study. BMC Health Serv Res 2018;18(1):878. doi: 10.1186/s12913-018-3715-4.

2. Harris RC, Grandjean L, Martin LJ, et al. The effect of early versus late treatment initiation after diagnosis on the outcomes of patients treated for multidrug-resistant tuberculosis: a systematic review. BMC Infect Dis 2016;16(1):193. doi:10.1186/s12879-016-1524-0.

3. Madala P, Jonnalagadda RK, Sastry TVSN, Samyukta R, Hanumanth $\mathrm{N}$, Raju CN. A study to identify the factors responsible for the delay in treatment initiation of MDR-TB cases registered under RNTCP in a district of South India. Int J Scient Res 2016;5(9):397-401. doi : 10.36106/ijsr.

4. Pan American Health Organization. Tuberculosis in the Americas, 2018. Washington, DC: PAHO, 2018 Document Number: PAHO/ CDE/18-036.

5. Htun YM, Khaing TMM, Aung NM, et al. Delay in treatment initiation and treatment outcomes among adult patients with multidrug-resistant tuberculosis at Yangon Regional Tuberculosis Centre, Myanmar: A retrospective study. PLoS One 2018;13(12): e0209932. doi: 10.1371/ journal.pone.0209932.

6. Htun YM, Khaing TMM, Yin Y, et al. Delay in diagnosis and treatment among adult multidrug resistant tuberculosis patients in Yangon Regional Tuberculosis Center, Myanmar: a cross-sectional study. BMC Health Serv Res 2018;18(1):878. doi: 10.1186/s12913-018-3715-4.

7. Zhang X, Yin J, Li H, et al. Diagnostic and treatment delays of multidrug-resistant tuberculosis before initiating treatment: a crosssectional study. Trop Med Int Health 2015;20(11):1431-1437. doi: 10.1111/tmi.12566

8. Rifat M, Hall J, Oldmeadow C, Husain A, Milton AH. Health system delay in treatment of multidrug resistant tuberculosis patients in Bangladesh. BMC Infect Dis 2015;15:526. doi: 10.1186/s12879-015-1253-9.

9. Okethwangu D, Birungi D, Biribawa C, et al. Multidrug-resistant tuberculosis outbreak associated with poor treatment adherence and delayed treatment: Arua District, Uganda, 2013-2017. BMC Infectious Diseases 2019;19(1):387. doi: 10.1186/s12879-019-4014-3.

10. Rifat M, Rusen ID, Islam MA, et al. Why are tuberculosis patients not treated earlier? A study of informal health practitioners in Bangladesh. Int J Tuberc Lung Dis 2011;15(5):647-651. doi: 10.5588/ijtld.10.0205.

11. World Health Organization. Automated real-time nucleic acid amplification technology for rapid and simultaneous detection of tuberculosis and rifampicin resistance: XpertMTB/RIF assay for the diagnosis of pulmonary and extrapulmonary TB in adults and children. Policy update. World Health Organization 2013. ISBN: 9789241506335.

Conflicto de intereses: Los autores declaran no tener conflicto de intereses. 